\title{
Salud electrónica (e-Salud): un marco conceptual de implementación en servicios de salud
}

\author{
Christian Díaz de León-Castañeda
}

Consejo Nacional de Ciencia y Tecnología (CONACYT), Centro de Investigación e Innovación en Tecnologías de la Información y Comunicación (INFOTEC), Aguascalientes, Aguascalientes, México.

\begin{abstract}
Resumen
La salud electrónica (e-Salud) es un concepto amplio que implica la aplicación de las tecnologías de la información y la comunicación en los sistemas de vigilancia, prevención, promoción y atención a la salud. Como componentes de la e-Salud se incluyen principalmente los sistemas de administración de insumos o recursos (agenda de citas, laboratorio clínico o farmacia), el expediente clínico electrónico, la prescripción electrónica, los sistemas de apoyo a la decisión clínica, el uso de dispositivos móviles, los sistemas de imagenología, los sistemas de atención a distancia, así como la enseñanza a través de medios digitales. Otros componentes que pueden considerarse parte de la e-Salud son los sistemas de almacenamiento y análisis masivo de datos, el aprendizaje artificial y los sistemas de "internet de las cosas". En este trabajo se propone un marco conceptual para analizar la implementación de componentes de e-Salud y sus efectos en la calidad de la provisión de servicios de salud.
\end{abstract}

PALABRAS CLAVE: Ciencia de la Información. Gestión de la calidad. Prestación de atención de salud. Recursos humanos en salud.

\begin{abstract}
Electronic health (e-Health) is a broad concept that involves the application of information and communication technologies to systems for surveillance, prevention, promotion and health care. e-Health components mainly include supply and resource management systems (appointment schedules, clinical laboratory or pharmacy), electronic medical records, electronic prescription, clinical decision support systems, use of mobile devices, imaging systems, remote care systems, as well as teaching through digital media. Other components that can be regarded as part of e-Health are the massive data storage and analysis systems, artificial learning, as well as "internet of things" systems. In this work, a conceptual framework is proposed in order to analyze the implementation of e-Health components and their effects on the quality of health services provision.
\end{abstract}

KEY WORDS: Information Science. Quality management. Healthcare provision. Human resources in health.

\section{Introducción}

La salud pública tiene dos grandes campos de estudio: las condiciones de salud de la población y los sistemas de salud como "respuesta social organizada" a estas condiciones. ${ }^{1} \mathrm{Al}$ respecto, para los sistemas de salud es necesario el continuo planteamiento de oportunidades que permitan mejorar el desarrollo de procesos y optimizar el uso de recursos en sus diferentes funciones básicas (rectoría, financiamiento, generación de recursos y provisión de servicios). ${ }^{2,3}$ En particular, en la función de provisión de servicios de salud es necesaria la continua mejora de la calidad de los procesos (preventivos, de promoción de la salud, curativos y rehabilitatorios). ${ }^{4,5}$

La calidad de la prestación de servicios de salud se ha definido como "el grado de ajuste entre las
Correspondencia:

Christian Díaz de León-Castañeda

E-mail: cddeleon@conacyt.mx
Fecha de recepción: 16-10-2017

Fecha de aceptación: 10-07-2018

DOI:10.24875/GMM.18003788
Gac Med Mex. 2019;155:176-183

Disponible en PubMed

www.gacetamedicademexico.com 
características de la oferta de los servicios y las expectativas de los usuarios o pacientes". Conceptualmente se reconocen dos grandes dimensiones para la evaluación de la calidad de la atención sanitaria: técnica e interpersonal. La dimensión técnica es "la aplicación de la ciencia y las tecnologías médicas de manera que rindan el máximo de beneficios para la salud sin aumentar con ello sus riesgos", mientras que la dimensión interpersonal es "el apego a valores y normas socialmente definidas que gobiernan la interacción de los individuos" y a los "dictados éticos de las profesiones relacionadas con la salud". ${ }^{6}$ Donabedian identificó tres grandes acercamientos en el análisis o evaluación de la calidad de la atención sanitaria: estructura, proceso y resultado, donde las características de estructura son determinantes de la calidad en el desarrollo de las actividades de proceso y a su vez estas de los resultados en salud. ${ }^{6}$ Asimismo, se han identificado diversas dimensiones que componen integralmente la calidad en la provisión de servicios de salud: la accesibilidad, la equidad, la efectividad, la eficiencia, la seguridad y la satisfacción. 4,5,7-10

Las tecnologías de la información y la comunicación (TIC) figuran como una oportunidad para la mejora de los diversos procesos relacionados con la salud. El vasto campo de aplicaciones de las TIC en el campo de la salud se conoce como salud electrónica ("e-Salud"), definida por la Organización Mundial de la Salud ${ }^{11}$ como "el uso coste-efectivo y seguro de las tecnologías de la información y comunicación (TIC) en apoyo a salud y a los ámbitos relacionados con la salud, incluyendo los servicios de atención sanitaria, vigilancia sanitaria, información en salud y educación, conocimiento e investigación en salud", sin embargo, el concepto de e-Salud aún se mantiene en debate y actualmente existen diversas conceptualizaciones. ${ }^{\text {12-14 }}$

La e-Salud integra un amplio grupo de aplicaciones (componentes de la e-Salud) que tienen la facultad de operar de forma integrada y pueden formar parte de diversos campos de dominio de la salud pública y los sistemas de salud (por ejemplo, vigilancia epidemiológica, prevención, promoción y atención a la salud en general). Algunos estudios han puesto de manifiesto la efectividad y eficiencia (costo-efectividad) de algunos componentes de la e-Salud..$^{15-18}$ Ante esta evidencia, la Organización Mundial de la Salud y otras instituciones - como la Organización Panamericana de la Salud, la Comisión Económica para América Latina y el Caribe y la Organización para la Cooperación y el Desarrollo Económicos- han emitido políticas encaminadas a la promoción de la implementación de TIC.19,20 Sin embargo, persisten problemas para el acceso, implementación y operación de estos componentes, principalmente en países en desarrollo, lo que genera brechas digitales en los niveles micro (entre personas), meso (entre instituciones $u$ organizaciones de salud) y macro (entre regiones geográficas o países). ${ }^{21,22}$

En este sentido, diversos estudios han puesto de manifiesto la necesidad de fortalecimiento de políticas de e-Salud, así como mejores sistemas de implementación de sus diversos componentes en los servicios de salud, de manera que se consideren los contextos sociales y operativos. ${ }^{23-25}$ En el campo tecnológico en salud, la ciencia de la implementación es una herramienta conceptual e instrumental que favorece el estudio de los factores que permiten optimizar la adopción y apropiación de tecnologías sanitarias basadas en evidencia científica (costo-efectivas); ;6,27 al respecto se han desarrollado diversos marcos analíticos. ${ }^{28,29}$

En este trabajo se presentan generalidades de los diversos componentes de la e-Salud y una breve sección del proceso de su adopción-apropiación en población en general y en personal sanitario. Estas bases se utilizan para presentar una propuesta del marco conceptual para el análisis de la implementación de los componentes de la e-Salud y su efecto en la calidad en la provisión de servicios de salud.

\section{Componentes de la e-Salud}

En la provisión de servicios de salud, las TIC figuran como recursos importantes para la conformación integral de los sistemas de información en salud $(\mathrm{SIS})^{\star}$ en sus diversos niveles de aplicación (poblacional o de servicios de salud). A través de estos sistemas se ha favorecido la comunicación entre profesionales sanitarios, entre profesionales sanitarios y pacientes, así como entre pacientes. A su vez, las TIC han penetrado en los sistemas de salud (por ejemplo, los sistemas de cómputo, la telefonía fija y celular o, más actualmente, los sistemas de comunicación basados en internet como el e-mail, las redes sociales,

\footnotetext{
* La Organización Mundial de la salud ha definido los SIS como "partes componentes interrelacionadas para la adquisición y análisis de datos y la provisión de información (información de administración, estadísticas de salud, bibliografía en salud) para el manejo de un programa o sistema sanitario y para el monitoreo de actividades en salud", ${ }^{30}$ así como un "esfuerzo integrado de acopio, procesamiento, notificación y uso de información y conocimientos sanitarios con objeto de influir en la formulación de políticas, la acción programática y las investigaciones". ${ }^{31}$
} 
los portales especializados, etcétera), lo que implica nuevos procesos de prevención, promoción y atención a la salud.

Las TIC han permitido mejores sistemas de almacenamiento y gestión de información a través de la digitalización de la documentación involucrada en los procesos administrativos como la información de los usuarios o pacientes, de los recursos (financieros, materiales y humanos) y de los insumos sanitarios en general (medicamentos, dispositivos médicos, entre otros). Específicamente destacan los sistemas de administración de servicios como la agenda de citas a los servicios de salud (cita electrónica a servicios médicos, odontológicos, de psicología, etcétera), así como los implicados con el laboratorio clínico y farmacia, entre otros. Asimismo, han evolucionado los procesos de atención sanitaria hacia la digitalización de la documentación, los expedientes médicos en papel pasan a ser expedientes clínicos electrónicos (ECE), ${ }^{32}$ las prescripciones médicas en papel pasan a ser prescripciones electrónicas, así como los resultados de estudios clínicos y de imagenología o radiología pasan de entregarse y analizarse en medios físicos hacia medios digitales (sistemas de comunicación y archivo de imágenes o PAC, picture archiving and communication systems). ${ }^{33}$

La evolución de las TIC ha permitido el desarrollo de sistemas de atención a distancia a través de medios electrónicos, desde el uso de sistemas de telefonía hasta basados en internet, lo que integra un amplio conjunto de sistemas que se engloban dentro del concepto de telesalud, que implica la prestación a distancia de servicios preventivos, de promoción de la salud o curativos. Un amplio campo dentro de la telesalud es la telemedicina y sus diversas especialidades de atención (por ejemplo, telerradiología, telepsiquiatría, telerrehabilitación, telecirugía, telecardiología, etcétera). Esta tecnología destaca por su potencial para favorecer el acceso a servicios de salud especializados para población de lugares remotos (por ejemplo, comunidades rurales, indígenas 0 poblaciones distantes). ${ }^{34-36}$

La evolución de las tecnologías de comunicación móviles como los teléfonos inteligentes (smartphones) y los dispositivos portátiles (wearables, como pulseras, relojes $u$ otros accesorios) permite la monitorización de actividades (ejercicio físico) o condiciones de salud para las personas y la monitorización de estas condiciones y estilos de vida por los profesionales sanitarios hacia la toma de decisiones. Estos dispositivos se aplican cada vez más en diversos campos y conforman la denominada "salud móvil" ("m-Salud" o "m-Health"). Desde una perspectiva de salud pública, estos dispositivos representan oportunidades de intervención a la población en general en promoción de la salud o de recopilación de información relacionada con los hábitos, conductas o estilos de vida de la población, lo que constituye una oportunidad para la emisión de posibles alertas de riesgo a la salud que puedan generar intervenciones oportunas (para los sistemas de vigilancia epidemiológica). ${ }^{37,38}$

Como parte de la e-Salud cabe destacar también los sistemas de apoyo a la decisión clínica (CDSS, clinical decision support systems), herramientas auxiliares a las que pueden recurrir principalmente los profesionales sanitarios para tomar decisiones durante la atención sanitaria, lo que implica un impacto potencial en la seguridad del paciente. Estos sistemas se han definido arbitrariamente como "sistemas de conocimiento activo que usan dos o más ítems de datos del paciente para generar consejo específico al caso", ${ }^{39}$ o como "software que es diseñado como una ayuda en la toma de decisiones clínicas en el que las características de un paciente individual se conjuntan con una base computarizada de conocimiento clínico, y entonces evaluaciones o recomendaciones específicas para el paciente son presentadas al personal clínico y/o al mismo paciente para una decisión", ${ }^{40}$ aunque se ha suscitado cierto debate y se ha sugerido ampliamente el concepto de "herramienta de decisión", que abarca otros sistemas de apoyo. ${ }^{41}$

Existe una amplia diversidad de tipologías en las que operan los CDSS, que depende de los contextos y del grado de implementación y desarrollo de los sistemas de TIC en los servicios de salud. Se ha propuesto una taxonomía para estos sistemas basada en cinco categorías: el contexto de uso, el recursos del conocimiento y datos, el tipo de apoyo a la decisión clínica, el tipo de entrega de la información y el flujo de trabajo u operación del sistema. ${ }^{42}$ En este campo se incluye el software basado en formularios o calculadoras para la toma de decisiones (por ejemplo, cálculos de depuración renal o escalas para la evaluación del estado de salud de los pacientes), así como el software

\footnotetext{
** La salud móvil o m-Salud es parte de la e-Salud y ha sido definida por el Observatorio Global para la e-Salud (de la Organización Munidad de la Salud) como "práctica médica y de salud pública apoyada por dispositivos móviles como teléfonos móviles, dispositivos para el monitoreo de pacientes, asistentes digitales personales (ADP) y otros dispositivos inalámbricos". Además, se identificaron las diferentes tecnologías de comunicación involucradas (SMS, GPRS, 3G, 4G, GPS, bluetooth). ${ }^{37}$
} 
especializado que identifica automáticamente errores durante la prescripción conforme las características del paciente.

Otro campo importante de la e-Salud es el uso de medios electrónicos o portales web para la educación en salud de profesionales sanitarios, usuarios de servicios y población en general ("e-learning"), con la finalidad de promover hábitos de buenas prácticas durante la atención sanitaria (personal sanitario), así como de promoción de la salud o hábitos saludables (población). Además, el uso de portales web es una oportunidad para facilitar a los pacientes el acceso a información de su salud, empoderándolos para su participación en las decisiones o para promover la comunicación con otros pacientes (junto con las redes sociales) y así conformar redes de apoyo, lo cual puede ser importante en algunos padecimientos crónicos (estas actividades se han relacionado con el término "e-paciente"). ${ }^{4 * * *}$

Como otras aplicaciones de las TIC en el área de la salud que están en desarrollo (o en uso en algunos países o sistemas de salud específicos) podrían mencionarse los siguientes:

- La gestión de almacenamiento, análisis y uso de grandes volúmenes de datos (big data) con propósitos de salud pública, vigilancia epidemiológica y promoción de la salud y mejora de la calidad en la provisión de servicios. ${ }^{45}$

- La aplicación de sistemas de "internet de las cosas" (IoT) con fines de monitorizar la salud y actividades de los pacientes (ya sea pacientes hospitalizados o ambulatorios, a través de dispositivos y sensores conectados a redes de internet), así como controlar su tratamiento (por ejemplo, la dosis de sus medicamentos). ${ }^{46.47}$

- Los sistemas de aprendizaje computacional ("machine learnig"), con fines de promover la automatización de procesos y servicios de atención, generando la capacidad ayuda en la toma de decisiones clínicas de diagnóstico o tratamiento de padecimientos. ${ }^{48,49}$

- El uso de tecnologías de realidad virtual o realidad aumentada con fines didácticos, preventivos o terapéuticos. ${ }^{50}$

- El desarrollo de la bioinformática y su aplicación en medicina (informática biomédica). ${ }^{51 \dagger}$

*** El término "e-paciente" se le atribuye a Ferguson, ${ }^{44}$ definido como "individuos equipados, habilitados, empoderados e involucrados en su salud y su atención en salud".

$\dagger$ La informática biomédica se refiere a la disposición de información génica de los pacientes para la toma de decisiones
- El continuo desarrollo de sistemas analíticos portátiles (dispositivos de análisis clínico o sensores) acoplados a sistemas informáticos móviles. ${ }^{52}$

Cabe destacar que los diferentes componentes de la e-Salud ofrecen la posibilidad de combinarse e interrelacionarse (interoperabilidad), generando un abanico de posibilidades que de forma integral representan oportunidades para fortalecer los sistemas de provisión de servicios de salud. ${ }^{53,54}$

\section{Adopción-apropiación de componentes de e-Salud}

Existen diversas formas en las que la sociedad puede adoptar y apropiarse ${ }^{\ddagger}$ de las TIC con fines de salud, identificándose dos principales tipos de actores: la población en general (incluyendo los usuarios de servicios de salud) y los prestadores de servicios de salud (ya sean profesionales o técnicos).

\section{Población en general y usuarios de servicios de servicios de salud}

De forma involuntaria, la población puede recibir información de prevención o promoción de la salud a través de medios de comunicación masiva como la televisión, la radio, mensajería SMS de teléfonos celulares 0 internet en sus diversas modalidades. Además, la población en general puede "adoptar" algunos componentes de e-Salud de forma voluntaria, por ejemplo, buscar información u orientación en salud a través del uso de medios como el teléfono (consulta vía telefónica) o internet. Esto requiere que las personas venzan las barreras (económicas o socioculturales) del acceso a estas tecnologías, tomando la decisión de adoptar estas tecnologías y adquirir las habilidades digitales necesarias para su uso.

En la adopción-apropiación de estas tecnologías por la población pueden influir diversos factores intrínsecos y extrínsecos. Entre los intrínsecos podrían jugar un papel la edad, el sexo, la ocupación, la escolaridad, el estrato socioeconómico y las condiciones

clínicas. Surge como un campo nuevo e interesante relacionado con la medicina genómica. ${ }^{51}$

‡ El término adopción alude al acto de decisión de usar las tecnologías (ya sea esta decisión tomada por personas u organizaciones). El término apropiación puede tener muchas connotaciones, niveles y dimensiones de análisis, a nivel individual hace referencia a un nivel de "maestría" en la incorporación de la tecnología en las actividades cotidianas y laborales. ${ }^{55,56}$ En la esfera profesional en salud se utiliza más ampliamente el término adopción. 
de salud de las personas; mientras que como factores extrínsecos pueden influir los círculos sociales a que se pertenece, la implementación de TIC en el lugar de trabajo, así como en las instituciones o servicios de salud donde se recibe atención y la influencia del personal sanitario que en ellas labora. Se han desarrollado diversos marcos teórico-conceptuales para el estudio y análisis de estos procesos. ${ }^{55-57}$

\section{Prestadores de servicios de salud}

La adopción y uso de algunos componentes de la e-Salud son exclusivos para personal sanitario, por ejemplo, el ECE, los sistemas de prescripción electrónica, los PAC, algunos CDSS, los sistemas de telesalud desde la parte prestadora del servicio, etcétera.

Al igual de los mencionados para la población en general, en el proceso de adopción-apropiación de estos componentes por el personal sanitario entran en juego factores intrínsecos como la edad, el sexo, la formación, el campo de la atención a la salud en que labora, así como factores extrínsecos como las prácticas adoptadas en el círculo profesional y la implementación de estos componentes en las instituciones o servicios de salud donde trabaja. En este sentido, para que el personal sanitario pueda adoptar estas tecnologías se necesita que las organizaciones de salud en las que laboran venzan las barreras de acceso y promuevan en su personal una adecuada implementación de estas tecnologías a través del desarrollo de habilidades o competencias digitales. Cabe destacar que algunos componentes pueden ser adoptados por el personal sanitario de forma voluntaria y autónoma, como las aplicaciones para teléfonos inteligentes o los portales web especializados que facilitan diversas tareas implicadas en la atención sanitaria. Se han desarrollado algunos modelos teórico-conceptuales para explicar el uso y satisfacción con estas tecnologías en el contexto organizacional. ${ }^{26-29,55-59}$

\section{Propuesta de marco conceptual}

Se ha planteado un marco para el estudio de la implementación de componentes de la e-Salud y su efecto en las dimensiones de análisis de la calidad en la provisión de servicios de salud identificadas por Donabedian y en otras revisiones conceptuales ${ }^{4-10}$ (Figura 1). Este marco conceptual involucra dos componentes principales que se revisan a continuación.

\section{Implementación de los componentes de e-Salud}

La implementación de tecnologías sanitarias se refiere a la acción de introducir estas tecnologías en la estructura o procesos que se llevan a cabo en los sistemas de provisión de servicios de salud. En particular, la ciencia de la implementación promueve el uso de tecnologías que han demostrado ser efectivas, eficientes y seguras (basadas en evidencia) ${ }^{\ddagger \ddagger}$ en la práctica operativa de los servicios de salud. ${ }^{26,27}$ Se han publicado diversos marcos analíticos y recomendaciones para realizar estudios de implementación. . $^{28,29,60,61}$

El primer paso para introducir los componentes de la e-Salud en los servicios de salud implica un proceso de análisis de factibilidad, que analiza los facilitadores y barreras para la implementación (de infraestructura, sociales o culturales). Este proceso debería estar fundamentado en estudios especializados que evalúen la necesidad de la implementación de la tecnología en el lugar o servicio propuesto, de manera que se promueva la equidad en el acceso a la intervención o tecnología a implementar. El siguiente paso es la "adopción organizacional", que implica la decisión de la organización de implementar la tecnología en sus procesos. Esta decisión puede estar influida por políticas externas e internas.

El tercer paso de implementación es la "adaptación", que implica el ajuste de los procesos internos de la organización (en este caso, procesos de atención a la salud), así como la capacitación de las personas (personal técnico o administrativo y los usuarios de servicios) hacia la introducción de las tecnologías en estos procesos, promoviendo la adquisición de las habilidades digitales necesarias. Durante este proceso de adaptación es necesaria la realización de "pilotajes" para analizar la operación de los procesos. Una evaluación o estudio importante es el de "fidelidad", que se refiere al estudio del grado en que una intervención fue implementada como fue previsto en determinado plan o protocolo de implementación (mientras que la evaluación de la adaptación se refiere al grado en que la estrategia o intervención es modificada por los usuarios o personas durante la implementación, para ajustarse a las necesidades particulares). ${ }^{26,60}$

拉 En el campo de la e-Salud la generación de evidencia científica sólida cobra importancia para evitar caer en el error de adoptar tecnologías con poca o nula evidencia. Asimismo, cobra importancia la generación de marcos normativos que promuevan este aspecto, así como el uso y operación en general de los componentes de e-Salud. 


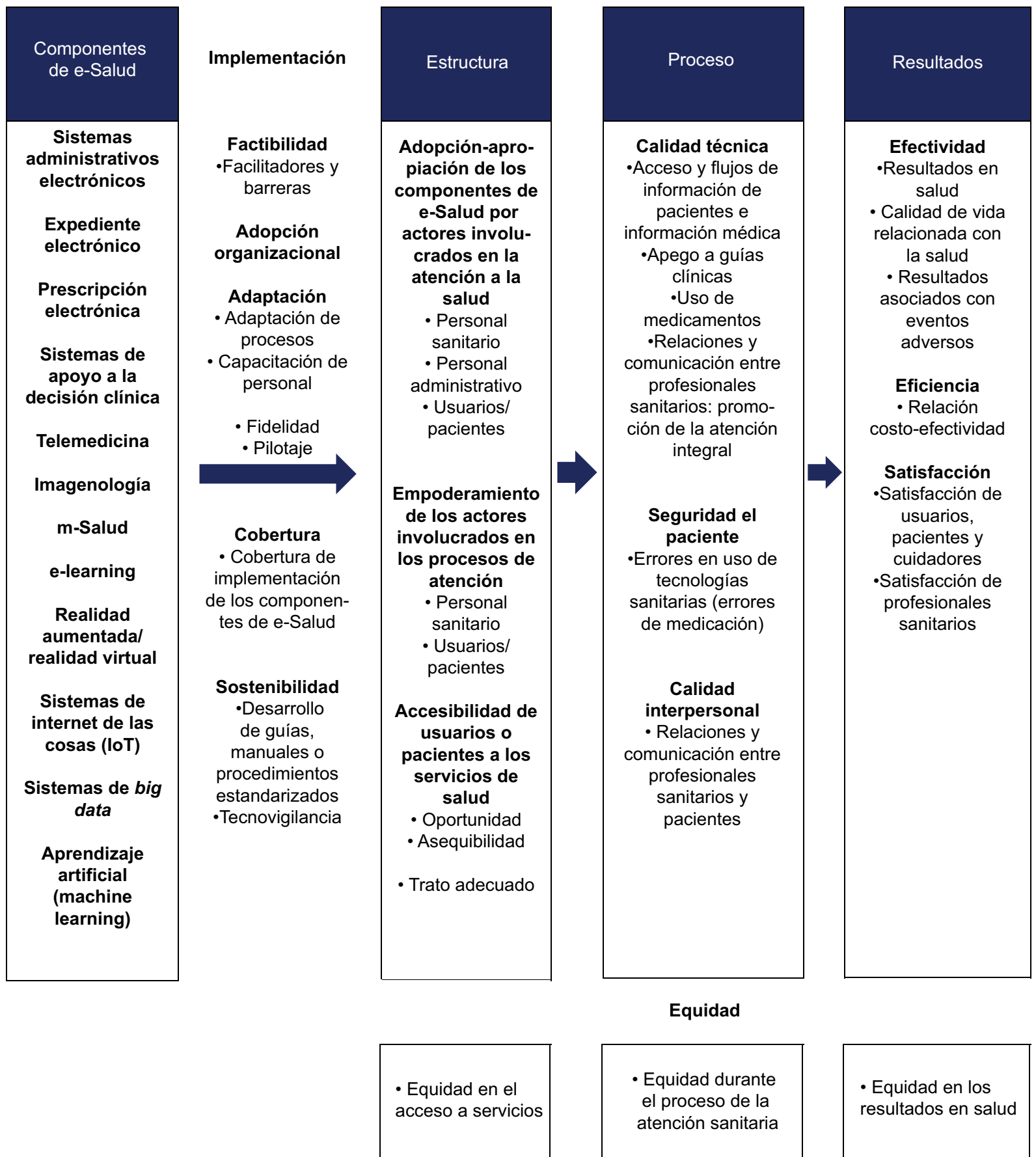

Figura 1. Marco conceptual para el análisis de la implementación de componentes de la e-Salud y su efecto en la calidad en la provisión de servicios de salud (elaboración propia).

El estudio de la adopción-apropiación tecnológica por los actores involucrados en los procesos de atención implica la investigación de la calidad en el uso de la tecnología y la satisfacción de estos actores con la misma. En el análisis e investigación de este proceso entran en juego los abordajes teórico-conceptuales mencionados.

Los estudios de "sostenibilidad" evalúan el grado en que una intervención o tecnología es mantenida o "institucionalizada" en la organización o servicio particular. ${ }^{26}$ Como parte de este aspecto podría incluirse el desarrollo de procedimientos normalizados de operación con fines de estandarizar o normalizar los procesos con base en los cambios que implica la introducción de TIC. En este punto, cabe destacar que es necesario el desarrollo de los procedimientos normalizados de operación o procedimientos especiales para vigilar el desempeño de las tecnologías, promoviendo los sistemas de tecnovigilancia. 
Finalmente, la "cobertura" es la evaluación de la fracción de población beneficiada con la implementación de la tecnología en relación con la elegible. ${ }^{26}$

\section{Análisis del efecto en la calidad en la provisión de servicios}

La implementación de los componentes de e-Salud en los servicios de salud genera un efecto o impacto en la calidad en la provisión de servicios de salud a diversos niveles:

a) Efectos en la estructura. En primer lugar, la implementación de los componentes de e-Salud en los servicios de salud mejorará la infraestructura tecnológica en TIC y en sus diversas modalidades, como las redes, telecomunicaciones, hardware y software. Además, la adopción de las TIC y el acceso a la información promueve el empoderamiento de los actores involucrados en los procesos de atención (tanto en personal sanitario como en usuarios 0 pacientes). Asimismo, algunos componentes de la e-Salud tienen la facultad de promover la accesibilidad a los servicios, ${ }^{9,10}$ como la cita electrónica y los portales electrónicos para pacientes, además de los sistemas de telesalud o telemedicina.

b) Efectos en el proceso. En este acercamiento se incluyen las dimensiones calidad técnica, calidad interpersonal y seguridad del paciente. En la dimensión calidad técnica, algunos componentes de la e-Salud podrían favorecer el acceso a sistemas de información de los usuarios o pacientes (o información médica en general) y el apego a guías clínicas, el uso adecuado de los medicamentos o la adherencia de los pacientes. Por su parte, algunos componentes de e-Salud podrían promover la seguridad del paciente, principalmente los CDSS (sistemas de vigilancia del apego a directrices o normas clínicas), con lo que constituirían una barrera que podría impedir que los errores en el uso de tecnologías sanitarias (como los medicamentos) alcancen al paciente. Por otra parte, las TIC podrían beneficiar la comunicación entre el personal sanitario, lo cual podría ayudar al trabajo colaborativo interprofesional (atención integral), que se considera un atributo importante en los procesos de prestación de servicios de salud. Asimismo, las TIC podrían promover la comunicación entre el personal sanitario y los pacientes o la adherencia de los pacientes a sus citas médicas o tratamientos (sistemas de recordatorios electrónicos). Como parte de la calidad interpersonal, algunos componentes de la e-Salud podrían favorecerla a través de mejorar la comunicación entre el personal sanitario y los usuarios o pacientes, sin embargo, también existe el riesgo de que la implementación de los componentes de e-Salud representen una barrera para la comunicación entre el personal sanitario y los pacientes, particularmente durante la consulta médica y en contextos de sobrecarga de trabajo.

c) Efectos en los resultados. Se incluyen las dimensiones efectividad, eficiencia y satisfacción. Dado que la efectividad es una consecuencia directa de los procesos o actividades que se realizan en el acercamiento de proceso, los componentes de la e-Salud tienen el potencial de impactar en los resultados en salud en las personas, así como su calidad de vida o promover un mejor uso de las tecnologías sanitarias que generaría mejores resultados asociados con su uso (por ejemplo, prevención de eventos adversos asociados con errores de medicación). Asimismo, el posible ahorro en el uso de recursos durante la atención sanitaria implicaría un impacto en la dimensión eficiencia. Además, la calidad interpersonal con la que se realizan los procesos de la atención sanitaria junto con los resultados en salud de los pacientes o la autopercepción de su salud de los mismos puede tener un impacto en su satisfacción.

d) Equidad. Los componentes de e-Salud figuran como una oportunidad de promover la equidad en salud en los niveles de estructura, proceso y resultado. Sin embargo, en este rubro se necesita promover la equidad en la implementación de TIC, de manera que los componentes de e-Salud no figuren como un elemento más de las desigualdades o inequidades en salud (brecha digital en salud).

\section{Conclusiones}

Las TIC son una herramienta valiosa e integral para la provisión de servicios de salud, que requieren el estudio continuo de su implementación e impacto en calidad, por lo que el marco conceptual propuesto puede ser un acercamiento en este rubro.

\section{Bibliografía}

1. Frenk J. La salud de la población, hacia una nueva salud pública. México: Fondo de Cultura Económica; 1994.

2. Murray C, Frenk J. A framework for assessing the performance of health systems. Bull World Health Organ. 2000;78:717-731.

3. World Health Organization. The world health report 2000. Health systems: improving performance. Ginebra, Suiza: World Health Organization; 2000. 
4. World Health Organization. Quality of care. A process for making strategic choices in health systems. Ginebra, Suiza: World Health Organization; 2006.

5. Institute of Medicine, Committee on Quality of Health Care in America. Crossing the quality chasm: a new health system for the $21^{\text {st }}$ century. BJM. 2001;323:1192

6. Donabedian A. La calidad de la atención médica, definición y métodos de evaluación. México: La Prensa Médica Mexicana; 1984

7. Arah OA, Westert GP, Hurst J, Klazinga NS. A conceptual framework for the OECD Health Care Quality Indicators Project. Int J Qual Health Care. 2006;18:5-13.

8. Campbell SM, Roland MO, Buetow SA. Defining quality of care. Soc Sci Med. 2000;51:1611-1625.

9. Frenk J. El concepto y la medición de accesibilidad. Salud Publica Mex. 1985;27:438-453.

10. Penchansky R, Thomas JW. The concept of access. Med Care. 1981;19:127-140.

11. World Health Organization. WHA58.28 e-Health. En: Fifty-eighth World Health Assembly (WHA58/2005/REC/1). Ginebra, Suiza: World Health Organization; 2005.

12. Boogerd EA, Arts T, Engelen LJ, Van-De-Belt TH. "What is eHealth": time for an update? JMIR Res Protoc. 2015;4:e29.

13. Eysenbach G. What is e-health? J Med Internet Res. 2001;3:e20.

14. Oh H, Rizo C, Enkin M, Jadad A. What is eHealth? A systematic review of published definitions. J Med Internet Res. 2005;7:1-12.

15. Elbert NJ, Van-Os-Medendorp H, Van-Renselaar W, Ekeland AG, Hakkaart-Van-Roijen L, Raat $\mathrm{H}$, et al. Effectiveness and cost-effectiveness of ehealth interventions in somatic diseases: a systematic review of systematic reviews and meta-analyses. J Med Internet Res. 2014; 16:1-23.

16. Flodgren G, Rachas A, Farmer A, Inzitari M, Shepperd S. Interactive telemedicine: effects on professional practice and health care outcomes. Cochrane Database Syst Rev 2015;9:CD002098.

17. Totten AM, Womack DM, Eden KB, McDonagh MS, Griffin JC, Grusing S, et al. Telehealth: mapping the evidence for patient outcomes from systematic reviews. Agency for Healthcare Research and Quality. 2016.

18. Iribarren SJ, Cato K, Falzon L, Stone PW. What is the economic evidence for mHealth? a systematic review of economic evaluations of mHealth solutions. PLoS One. 2017;12:e0170581.

19. World Health Organization. eHealth. Report by the Secretariat. Ginebra, Suiza: World Health Organization; 2004.

20. Organización Panamericana de la Salud. Estrategia y plan de acción sobre eSalud. EE. UU.: Organización Panamericana de la Salud; 2011.

21. World Health Organization. Atlas of eHealth country profiles 2015: the use of eHealth in support of universal health coverage. Based on the findings of the third global survey on eHealth 2015. Ginebra, Suiza: World Health Organization; 2016.

22. Lewis T, Synowiec C, Lagomarsino G, Schweitzer J. E-health in low- and middle-income countries: findings from the Center for Health Market Innovations. Bull World Health Organ. 2012;90:332-340.

23. Lau F, Kuziemsky C, Price M, Gardner J. A review on systematic reviews of health information system studies. J Am Med Informatics Assoc. 2010; 17:637-645.

24. Car J, Black AD, Anandan C, Pagliari C, Anandan C, Cresswell K, et al. The impact of eHealth on the quality \& safety of health care: a systematic overview. PLoS One. 2011;8:e1000387.

25. Socio-economic impact of mHealth. An assessment report for Brazil and Mexico. India: PricewaterhouseCoopers Private Limited; 2013.

26. Peters DH, Adam T, Alonge O, Agyepong IA, Tran N. Implementation research: what it is and how to do it. BMJ. 2013;347:f6753.

27. Spiegelman D. Evaluating public health interventions: 1. Examples, definitions, and a personal note. Am J Public Health. 2016;106:70-73.

28. Nilsen P. Making sense of implementation theories, models and frameworks. Implement Sci. 2015;10(1):1-13.

29. Tabak RG, Khoong EC, Chambers D, Brownson RC. Bridging research and practice: models for dissemination and implementation research. Am J Prev Med. 2012; 43(3):337-350.

30. World Health Organization. Health information systems development and strengthening. Guidance on needs assessment for national health information development. Ginebra, Ginebra, Suiza: World Health Organization; 2000.

31. World Health Organization. The world health report 2003. Shaping the future. Ginebra, Suiza: World Health Organization; 2003.

32. Heart T, Ben-Assuli O, Shabtai I. A review of PHR, EMR and EHR integration: a more personalized healthcare and public health policy. Health Policy Technol. 2017;6:20-25.
33. Van-De-Wetering R, Batenburg R, Versendaal J, Lederman R, Firth L A balanced evaluation perspective: picture archiving and communication system impacts on hospital workflow. J Digit Imaging. 2006; 19:10-17.

34. Van-Dyk L. A review of telehealth service implementation frameworks. Int J Environ Res Public Health. 2014;11:1279-1298.

35. Scott R, Mars M. Telehealth in the developing world: current status and future prospects. Smart Homecare Technol TeleHealth. 2015;3:25-37.

36. Wootton R, Patil NG, Scott RE, Ho K. Telehealth in the developing world. Inglaterra: The Royal Society of Medicine Press; 2009.

37. World Health Organization. mHealth. New horizons for health through mobile technologies. Ginebra, Suiza: World Health Organization; 2011.

38. Malvey D, Slovensky DJ. mHealth transforming healthcare. EE. UU.: Springer Science; 2015.

39. Wyatt J, Spiegelhalter D. Field trials of medical decision-aids: potential problems and solutions. Proc Annu Symp Comput Appl Med Care. 1991;3-7.

40. Sim I, Gorman P, Greenes RA, Haynes RB, Kaplan B, Lehmann H, et al. Clinical decision support systems for the practice of evidence-based medicine. J Am Med Inf Assoc. 2001;8:527-534.

41. Liu J, Wyatt JC, Altman DG. Decision tools in health care: focus on the problem, not the solution. BMC Med Inform Decis Mak. 2006:6:4.

42. Berlin A, Sorani M, Sim I. A taxonomic description of computer-based clinical decision support systems. J Biomed Inform. 2006;39:656-667.

43. Eysenbach G. Medicine 2.0: social networking, collaboration, participation, apomediation, and openness. J Med Internet Res. 2008;10:e22

44. Riggare S. E-patients hold key to the future of healthcare. BMJ. 2018;360:k846.

45. Raghupathi W, Raghupathi V. Big data analytics in healthcare: promise and potential. Health Inf Sci Syst. 2014;2:3.

46. Dimitrov DV. Medical internet of things and big data in healthcare. Health Inform Res. 2016;22:156-163.

47. Yin $Y$, Zeng $Y$, Chen $X$, Fan $Y$. The internet of things in healthcare: an overview. J Ind Inf Integr. 2016;1:3-13.

48. Clifton DA, Niehaus KE, Charlton P, Colopy GW. Health informatics via machine learning for the clinical management of patients. Yearbook Med Inform. 2015;10:38-43.

49. Jha S, Topol EJ. Adapting to artificial intelligence: radiologists and pathologists as information specialists. JAMA. 2016;316:2353-2354.

50. Mirelman A, Rochester L, Maidan I, Del-Din S, Alcock L, Nieuwhof F, et al. Addition of a non-immersive virtual reality component to treadmill training to reduce fall risk in older adults (V-TIME): a randomised controlled trial. Lancet. 2016;388:1170-1182.

51. Martin-Sanchez F, lakovidis I, Nørager S, Maojo V, De-Groen P, VanDer-Lei J, et al. Synergy between medical informatics and bioinformatics: facilitating genomic medicine for future health care. J Biomed Inform. 2004;37:30-42.

52. Radin JM, Topol EJ, Andersen KG, Steinhubl SR, Brendish N, Schiff H, et al. A laboratory in your pocket. Lancet. 2016;388:1875.

53. Indarte S, Pazos-Gutiérrez P. Estándares e interoperabilidad en salud electrónica: requisitos para una gestión sanitaria efectiva y eficiente. Chile: Comisión Económica para América Latina y el Caribe; 2011.

54. Adebesin F, Kotzé P, Ritz D, Foster R, Van-Greunen D. Method for selecting e-health standards to support interoperability of healthcare information systems. En: IST-Africa 2014 Conference Proceedings. Tanzania: International School of Tanganyika; 2014.

55. Fernández-Morales K, Vallejo-Casarín A, McAnally-Salas L. Apropiación tecnológica: una visión desde los modelos y las teorías que la explican. Perspect Educ. 2015;54:109-125.

56. Isaac $\mathrm{H}$, Besseyre-Des-Horts $\mathrm{CH}$, Leclercq $\mathrm{A}$. Adoption and appropriation: towards a new theoretical framework, an exploratory research on mobile technologies in French companies. Systèmes d'Information Manag. 2006;11:9-50.

57. Korpelainen K. Theories of ICT system implementation and adoption: a critical review. Finlandia: School of Science and Technology/Aalto University; 2011.

58. Delone $W H$, McLean $E$. The DeLone and McLean model of information systems success: a ten-year update. J Manag Inf Syst. 2003;19:9-30.

59. Cooper RB, Zmud RW. Information technology implementation research: a technological diffusion approach. Manag Sci. 1990;36:123-139.

60. Pinnock H, Barwick M, Carpenter CR, Eldridge S, Grandes G, Griffiths CJ, et al. Standards for Reporting Implementation Studies (StaRI) Statement. BMJ. 2017;356:i6795.

61. Wilson PM, Sales A, Wensing M, Aarons GA, Flottorp S, Glidewell L, et al. Enhancing the reporting of implementation research. Implement Sci. $2017 ; 12: 13$. 